\title{
Tecnologias em Sistemas Interativos e Inteligentes para Ensino Virtual
}

\author{
Liliane S. Machado, Ronei M. Moraes \\ Laboratório de Tecnologias para o Ensino Virtual e Estatística (LabTEVE) \\ Universidade Federal da Paraíba - CCEN \\ 58051-900 - João Pessoa - PB - Brasil \\ liliane@di.ufpb.br, ronei@de.ufpb.br
}

O grupo de Tecnologias em Sistemas Interativos e Inteligentes para Ensino Virtual desenvolve pesquisas de cunho multi e interdisciplinar e está vinculado à Universidade Federal da Paraíba (UFPB). O grupo foi criado no ano 2000 com o intuito de desenvolver e integrar tecnologias voltadas ao Ensino Virtual e ao Ensino a Distância. Neste contexto, está incluída a pesquisa e uso de diversas metodologias com o objetivo promover e apoiar o aprendizado por meio de sistemas locais ou de acesso remoto. Uma vez que as soluções visam o apoio ao ensino nas diversas áreas das ciências, estas englobam conceitos que vão da estatística às ciências da computação, matemática, saúde e engenharia, dentre outras áreas, além da educação. Algumas das linhas de pesquisa do grupo são a realidade virtual, serious games, interação gráfica e háptica, educação colaborativa e educação à distância. $\mathrm{O}$ grupo desenvolve projetos científicos, tecnológicos e de inovação, cujos projetos estão inseridos nos programas de pósgraduação em Modelos de Decisão em Saúde e em Informática da Universidade Federal da Paraíba. Mais detalhes podem ser encontrados em http://www.de.ufpb.br/ labteve.

1) Universidades e Instituições de Pesquisa parceiras:

- Laboratório Nacional de Computação Científica (LNCC-Brasil)

- State University of New York (SUNY-OSWEGO - EUA)

- Universidade do Porto (Portugal)

- Université Toulouse III - Paul Sabatier (IRIT - França)

2) Pesquisadores envolvidos

Liliane Machado, Ronei Moraes, Thaise Costa, Luana Almeida, Paulo Paiva,

Douglas Ferreira, Elaine Soares, Ives Moura, José Segundo, Thiago Batista,

Carlos Xavier, Edviges Lima, Ana T. Medeiros, Ana M. Valença, Ulisses Anjos.

3) Principais projetos (e fomentos recebidos)

Ingá Virtual - ambiente virtual 3D do sítio arqueológico Pedra do Ingá (MinC)

Museu 3I - museu virtual tridimensional configurável e online (MinC)

CyberMed - framework para desenvolvimento de sistemas de realidade virtual para treinamento e avaliação de habilidades médicas $(\mathrm{CNPq})$

Calculadora Estatística -ferramenta para suporte ao ensino de Estatística (CNPq)

Laboratórios Remotos Táteis - ferramenta online para suporte ao aprendizado de engenharia mecânica

SilvesterPEC - serious game para apoio ao aprendizado de Geografia (FINEP)

GeoPlanoPEC, GeoEspaçoPEC e GeoPlanoMOB - serious games para apoio ao aprendizado de matemática no $1^{\circ}$ grau (FINEP)

Caixa de Pandora - serious game para capacitação de profissionais de saúde sobre violência contra a mulher (CAPES/CNPq)

Treinamento Colaborativo de Equipes - sistema de realidade virtual e serious games para capacitação em saúde (INCT-MACC, CNPq e CAPES) 